\title{
ON THE REGIONS OVER SUNSPOTS AS STUDIED BY POLARIZATION OBSERVATIONS ON CENTIMETER WAVELENGTHS
}

\author{
G. GELFREICH, D. KOROL'KOV, N. RISHKOV, AND N. SOBOLEVA \\ Pulkovo Observatory, Leningrad, U.S.S.R.
}

The observations of the sun at centimeter wavelengths made at Pulkovo in 1956-58 have shown: (1) There are regions of appreciable size over the majority of sunspots that have "enhanced" radio emission at centimeter wavelengths [1]. The nature of this emission shows that it belongs to the slowly varying component. In fact, as long as a group of sunspots persists, the flux of such an active region preserves its almost constant value. (2) The emission is partly circular, the polarized flux changing in magnitude but inappreciably. The sign of polarization rotation remains constant [2], which appears to be positive proof that a rather intense and stable magnetic field exists in these regions. (3) The extent of the regions is about that of the spot nuclei [3]. (4) Their brightness temperature amounts to several million degrees. (5) The height at which enhanced radio emission is produced is of the order $1.07 \pm 0.02 R_{\odot}[4]$.

Considering the above, we have to do with thermal emission from some dense, hot regions in the lower corona over sunspots (the so-called coronal condensations). These regions seem to have an intense magnetic field. Up to the present time, it has been possible to measure the magnetic field over sunspots only to a height of 2000 to $2500 \mathrm{~km}$ above the photosphere [5]. Determining the magnetic field in the upper chromosphere by optical methods is altogether impossible.

To investigate the physical conditions in the regions over the spots by radio observations one must know their size and brightness temperatures (both polarized and unpolarized) over a sufficiently wide range of wavelengths. Suppose we have derived from observations the dependence of brightness temperature of condensation on frequency. The brightness temperature over a radio range, where the Rayleigh-Jeans law holds, is computed by the formula

$$
T_{b}=\int_{0}^{\tau} T_{k} \exp (-\tau) d \tau
$$

and provided $T_{k}=$ const.,

$$
T_{b}=T_{k}[1-\exp (-\tau)]
$$

where $T_{k}$ is the kinetic temperature, and $\tau=\int \kappa d s$ is the optical thickness. 


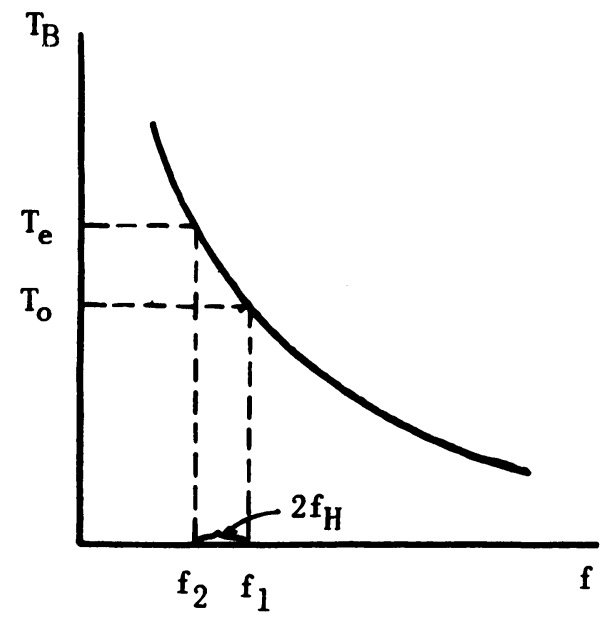

Fig. 1. Determination of magnetic field.

If the magnetic field is absent, the absorption coefficient $\boldsymbol{k}$ due to free-free transitions is proportional to $1 / f^{2}$, where $f$ is the emission frequency. In case the magnetic field is present, the electromagnetic wave is split into two normal waves-one ordinary and one extraordinary. At this the coefficient of absorption changes, becoming proportional to $\left(f+f_{B}\right)^{-2}$ and $\left(f-f_{B}\right)^{-2}$ for the ordinary and extraordinary waves respectively, $f_{H}=e H / 2 \pi m c, H$ being the magnetic field [6]. Thus, the transfer equation (1) gives the same brightness temperature as would be expected in the absence of magnetic field for frequencies $f_{1}=f+f_{H}$ and $f_{2}=f-f_{H}$. Such is the case when the refraction index $n_{1,2}$ differs but little from unity. Suppose we derived from observations the dependence of brightness temperature of a condensation on frequency. Once the value of brightness temperature for the ordinary and extraordinary waves is known, the value of $f_{1}-f_{2}=2 f_{H}$ and, consequently, that of the magnetic field can be determined (Fig. 1).

The value of the kinetic temperature and electron density can be derived as follows. Suppose the emission region to be semitransparent for the waves $\lambda_{1}$ and $\lambda_{2}$. By our observing over a sufficiently wide wavelength range we can find such waves, for which the expression

$$
P_{\lambda_{i}}=\frac{2 k T_{k}\left[1-\exp \left(-\tau_{i}\right)\right] \Omega}{\lambda_{i}{ }^{2}}=\frac{2 k T_{k}\left(\tau_{i}-\frac{1}{2} \tau_{i}{ }^{2}\right) \Omega}{\lambda_{i}{ }^{2}}
$$

holds true. Here $P_{\lambda}=$ flux density; $k=$ Boltzmann's constant; $T_{k}=$ kinetic temperature; $\Omega=$ solid angle of the emitting region; $\tau_{i}$ (optical thickness) $=$ $\tau_{0} \lambda_{t}^{2} ;$ and $\tau_{0}=f\left(N_{e}, T_{k}\right)$, where $N_{e}=$ the electron density.

Using the flux for waves $\lambda_{1}$ and $\lambda_{2}$ the following equation is obtained

$$
\frac{P_{\lambda_{1}}^{2}}{P_{\lambda_{1}}-P_{\lambda_{2}}}=\frac{4 k T_{k} \Omega}{\left(\lambda_{2}^{2}-\lambda_{1}^{2}\right)}
$$

from which the kinetic temperature is found; whereupon the electron density is derived from (2).

To determine the above parameters, an eclipse expedition was sent to the Chinese People's Republic (Hainan Island) in 1958 April. The expedition from Pulkovo was equipped with three polarization instruments for the observations on 5.1 , and 3.3 , and $2.0-\mathrm{cm}$ wavelengths.

Fig. 2 represents the variation curve of the general radio-emission flux of 
the sun during the eclipse at $5.1 \mathrm{~cm}$. Two details can be clearly seen on this curve: one, the overlapping of a group of sunspots $\left(2^{\mathrm{h}} 04^{\mathrm{m}}-2^{\mathrm{n}} 12^{\mathrm{m}} \cdot 5\right.$ U.T.); the other, the sun's limb. Fig. 3 gives the records of the circularly polarized flux at 5.1 -and $3.3-\mathrm{cm}$ wavelengths at the times when the sunspot group is occulted and uncovered. The curves show several portions in which the derivative is changed. Since the difference between the

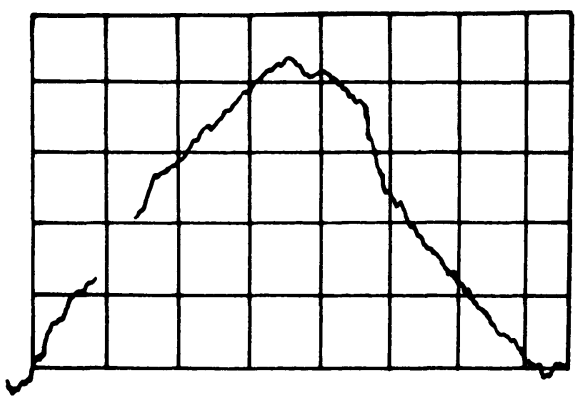

FIG. 2. Variation of $5.1-\mathrm{cm}$ radiation. inclinations of the moon's limb at the times of uncovery and the occultation of the sunspot group amounted to about $40^{\circ}$, the emitting region can be determined with some certainty.
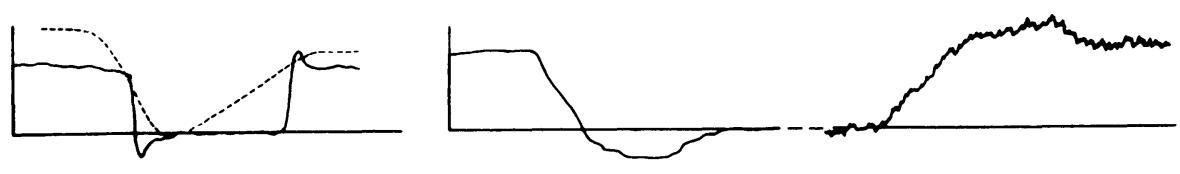

$3.3 \mathrm{~cm}$
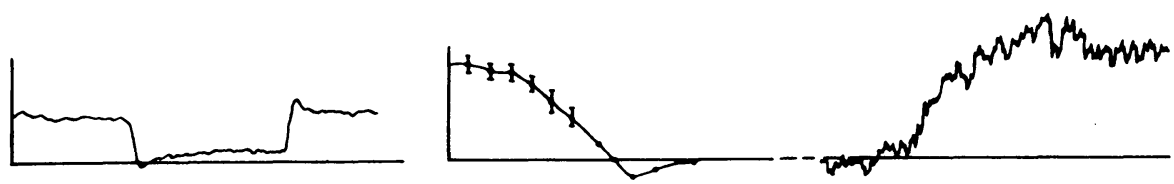

$5.1 \mathrm{~cm}$

Fig. 3. Circularly polarized flux at $5.1 \mathrm{~cm}$ (below) and $3.3 \mathrm{~cm}$ (above).

Fig. 4 represents a group of spots and a region giving polarized emission at 3.3 and $5.1-\mathrm{cm}$ wavelengths. The difference in the dimensions of the emitting regions obtained at these wavelengths is probably due to the records at $5.1 \mathrm{~cm}$, which are less certain because of the lower sensitivity of the apparatus. The regions above the two large nuclei of the group, extending over an area of 0.4 square minutes of arc, gave a left-hand polarized emission; the following spot gave right-hand polarized emission.

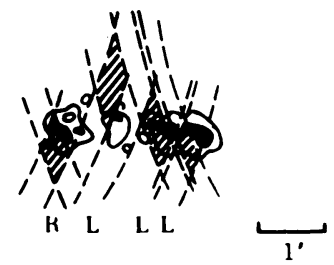

$3.3 \mathrm{~cm}$

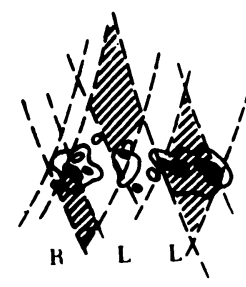

$5.1 \mathrm{~cm}$

Fig. 4. A polarized spot group at $3.3 \mathrm{~cm}$ (left) and $5.1 \mathrm{~cm}($ right).

It will be noted here that according to optical data in the photosphere the value of the magnetic field of large nuclei is 2400 gauss $S$, while over the following spot it is $1800 \mathrm{~N}$. If we assume that the sizes of the regions emitting both at 5.1 and $3.3-\mathrm{cm}$ wavelengths are the same, and that the dimensions of the regions giving off polarized and unpolarized emission coincide as well, then the brightness 
temperature of the emitting region can be found from the above curves at different wavelengths. The results are given in Table I.

TABLE I

$\begin{array}{ccc}\begin{array}{c}\text { Wavelength } \\ \mathrm{cm}\end{array} & \begin{array}{c}\text { Unpolarized } \\ { }^{\circ} \mathrm{K}\end{array} & \begin{array}{c}\text { Polarized } \\ { }^{\circ} \mathrm{K}\end{array} \\ 5.1 & 3.6 \times 10^{8} & 0.775 \times 10^{8} \\ 3.3 & 1.65 \times 10^{8} & 350 \times 10^{3} \\ 2.0 & <200 \times 10^{3} & <120 \times 10^{3}\end{array}$

The unpolarized brightness temperature has been taken from observations made with the large radiotelescope at Pulkovo. At the $2.0-\mathrm{cm}$ wavelength the emission of the region appeared to be below the sensitivity of the equipment.

These data were used for determining the magnetic field, the kinetic temperature, and the electron density by the method described above. The magnetic-field strength was found $\simeq 360$ gauss, the kinetic temperature $-7 \pm 3 \times 10^{6}{ }^{\circ} \mathrm{K}$, the emission measure $\int N_{e}^{2} d s=2.38 \times 10^{30}$.

If we assume that the region emitting the polarized flux is distributed precisely over the spot nuclei, we find that the height is $1.05 R_{\odot}$, or $35,000 \mathrm{~km}$ above the level of the photosphere. Our data are not sufficient to make definitive conclusions; hence, our results should be considered as preliminary.

Besides measuring the circular polarization, we attempted also during the eclipse to reveal linear polarization from the group of sunspots at $3.3 \mathrm{~cm}$. However, the linearly polarized flux was found to be below the threshold of sensitivity $\left(0.5 \times 10^{-22}\right.$ watts $\left.\mathrm{m}^{-2}(\mathrm{c} / \mathrm{s})^{-1}\right)$ (Fig. 2 dotted curve).

The work on polarization of radio emission from the sun is one of a number of investigations carried on in the Department of Radio Astronomy of the Pulkovo Observatory under the supervision of Professor S. E. Khaikin. The authors take this opportunity to thank him for his guidance and valuable suggestions. They also wish to express their gratitude to the Chinese scientists who participated in the eclipse observations under the direction of Professor Chen Fan-yun and greatly aided in securing the data used in this paper.

\section{REFERENCES}

[1] Ikhsanova, V. N. Izv. Obs. Pulkovo, 162, 1958 (in press).

[2] Kaìdanovskii, N. L., Korol'kov, D. V., Soboleva, N. S., and Khaikin, S. E. Dokl. Akad. Nauk S.S.S. R. 112, 1012, 1957.

[3] Korol'kov, D. V., and Soboleva, N. S. Solar Data, Moscow, 1, 1957.

[4] Ikhsanova, V. N. Solar Data, Moscow, 6, 1957.

[5] Severnyi, A., and Bumba, V. The Observatory, 78, 902, 1958.

[6] Al'pert, Ia. L., Ginzburg V. L., and Fainberg, E. L. Propagation of Radiowaves. Moscow, 1953. 